\title{
Phase Equilibria Modification by Electric Fields
}

\author{
Oak Ridge National Laboratory
}

September 1997

\section{Progress Report}

\section{Principal Investigator}

C. Tsouris

(423) 241-3246 (Phone)

(423) 241-4829 (Fax)

tsourisc@ornl.gov

Oak Ridge National Laboratory

P.O. Box 2008

Oak Ridge, TN 37831-6226

\section{Co-Investigator}

V.M. Shah

University of Tennessee

(610) 992-1480 (Phone)

shahv@ibm.net

618 Caley Road

King of Prussia, PA 19406-1555

\section{Contributors}
K. D. Blankenship, ORNL
M. A. Spurrier, ORNL
S. S. Laughlin, ORNL 


\section{Research Objectives}

In this research program, Oak Ridge National Laboratory is investigating the modification of phase equilibria and interface transport enhancement-caused by electric fields. The majority of environmental and waste treatment processes involve complex chemical separations and reactions. The treatment efficiency in such processes is governed by thermodynamic equilibria and transport. The objective of this project is to use electric fields to favorably manipulate the thermodynamic and transport properties of mixtures so that higher separation efficiencies can be achieved. An understanding of the mechanisms of the underlying phenomena of molecular and fluid interactions with electric fields will lead to the development of efficient remediation methods for contaminated natural environments and wastes.

\section{Research Statement}

The main focus of this project is to understand and quantify the influence of electric fields on thermodynamic equilibria and transport properties of fluid mixtures and to determine the conditions and properties of the systems for which this influence is of practical significance. The specific objectives of the project are discussed in the following paragraphs.

- Vapor-Liquid Equilibria (VLE). In previous works, it has been reported that electric fields can be used to enhance mass transport in multiphase systems. Because the extent of mass transport is limited by thermodynamic equilibria, the use of electric fields to change phase equilibria is being investigated in this project. An apparatus has been designed and constructed for conducting VIE experiments under electric fields. A study of the effect of electric fields on thermodynamic equilibria and transport of polar-polar, polar-nonpolar, and nonpolar-nonpolar mixtures is currently under way.

- Liquid-Liquid Equilibria (LLE). In this project, the LLE studies are conducted using biphasic, binary/ternary, liquid mixtures. An apparatus has been designed and constructed for LLE experiments under electric fields. The effect of electric fields on thermodynamic equilibria and transport of several immiscible or partially miscible mixtures, including aqueous-organic and aqueous-solute-organic, is being investigated.

- Investigation of the Mechanism. It is expected that the mechanism of phase equilibria modification is linked to such properties of the mixtures as polarity of the molecules, hydrogen bonding, and cluster formation. In this project, we are studying nonlinear dielectric effects using dielectric spectroscopy, as well as molecular interactions under electric fields using infrared (IR) and Raman spectroscopy.

- Interfacial. Transport Effects of Electric Fields. This research involves multiphase systems. It has been found that electric fields cause various interfacial phenomena that are important in determining equilibria and transport between various phases. Some of these phenomena are being investigated in this project. 
- Solid-Liquid Equilibria (SLE) and Solid-Vapor Equilibria (WE). Studies of SLE and SVE modification by electric fields, as related to environmental waste treatment processes, are included in this project.

\section{Research Progress}

In FY 1997, our studies have been focused on VLE and transport properties of mixtures. A number of novel experimental arrangements have been developed for various studies that were initiated in FY 1997 and are planned to be continued in FY 1998 and FY 1999. The progress made thus far is summarized.

\section{VLE and Batch Distillation Studies}

Specially designed VLE and batch distillation apparatuses have been constructed to allow accurate monitoring and control of the heating rate, pressure, and temperature. In addition to these monitoring devices, which are connected to the VLE and distillation units, high-voltage electrodes are introduced across the vapor-liquid interface where, upon application of a high voltage, an electric field is formed. The applied voltage is kept constant, and the electric current is monitored. Extensive experimental work has been done on VLE modification by electric fields.

In parallel to the VLE studies, batch distillation experiments have also been conducted using the same mixtures. The concentrations of the vapor and liquid mixtures were determined by gas chromatography. The mixtures used thus far are classified as nonpolar-polar, polar-polar, and nonpolar-nonpolar. Included in these systems are toluene-isopropanol, toluene-cyclohexane, isopropanol-water, cyclohexane-methyl isobutyl ketone (MIBK) and n-heptane-acetyl acetone.

Results showed that the electric field does not affect the composition of the vapor or liquid phase of a nonpolar-nonpolar system. It does, however, affect the vapor composition for polarnonpolar and polar-polar systems. It was observed that at high voltage (on the order of $20 \mathrm{kV}$ ), the electric field impedes the separation of the components, while at lower voltage (on the order of $10 \mathrm{kV}$ ), the electric field enhances the separation factor up to $10 \%$. It is believed that at $20 \mathrm{kV}$, the small droplets that are formed at the interface are entrained by the vapor, thereby decreasing the separation factor. This hypothesis is supported by visual observations of drop formation and spraying at the surface of the electrode that is located in the vapor phase as well as measurements of the current. It was found that the electric current at $20 \mathrm{kV}$ is much higher than at $10 \mathrm{kV}$. The separation factor increases when the voltage is high and the current is kept low. Once a highvoltage, low-current condition is applied following a no-voltage equilibrium condition, the vapor temperature decreases as "much as $1{ }^{\circ} \mathrm{C}$.

This temperature drop, which is measured by a high-accuracy thermometer, is consistent in all experiments under similar conditions. High-accuracy pressure measurements during the temperature drop are not yet available. It is believed that both temperature and pressure, as well as the interfacial concentration and properties, play significant roles in this phenomenon. Experiments to better understand the relationship involved are planned in FY 1998. A special cell has also been constructed for vapor-pressure measurements under electric fields. This effort will be completed in FY 1998. 


\section{LLE Studies}

A specially designed LLE cell that allows the introduction of electrodes across the liquidliquid interface, as well as temperature monitoring and control, has been constructed. Various binary and ternary liquid systems, including cyclohexane-water, cyclohexane-isopropanolwater, tributyl phosphate-water, and water-MIBK-acetic acid, have been used in this project. Early in the experiments, it was found that high voltage in liquid systems, in general, is associated with high levels of electric current and heat generation. Another limitation that was found is that the interface becomes unstable at high voltage, forming droplets and bridges between the two electrodes. Because of such limitations, at this point there are no conclusive results on LLE modification by electric fields. Better-controlled studies are planned for FY 1998.

\section{Investigation of the Mechanism}

Preparative work on the investigation of the mechanism of VLE modification by electric fields has been conducted in FY 1997 by using Raman, IR, and dielectric spectroscopy. Special equipment arrangements have been set up for these spectroscopic methods, and some preliminary experiments have been conducted with Raman and IR spectroscopy. Raman spectra of the toluene-isopropanol mixture did not show conclusive results on hydrogen bonding enhancement under an electric field. A cell has been designed and constructed for flow-through IRspectroscopy measurements under electric fields. Results from preliminary experiments have indicated that the thickness of the cell needs to be decreased before meaningful measurements can be obtained. More systematic experiments with a corrected cell are planned for FY 1998.

In addition, a high-frequency oscilloscope operating in the range of $\mathbf{0 . 5 - 2 0 ~} \mathbf{~ G H z}$ has been purchased, and an experimental setup for dielectric spectroscopy measurements under electric fields is being assembled. The key issue in this setup involves the design and construction of the experimental cell. This task, which will be completed in FY 1998, will allow us to study the mechanism of the effect of electric fields on phase equilibria modification.

\section{Interfacial Transport Effects of Electric Fields}

Electrohydrodynamic interfacial and bulk effects of the electric fields were also investigated. The spraying of air in water and the formation of microbubbles were extensively studied. Three modes of air spraying were identified: spraying, intermediate spraying-dripping, and dripping. The bubble size was measured under spraying conditions and found to be in the range of 10-70 $\mu \mathrm{m}$. It was also shown that electric fields cause simultaneous pumping, spraying, and mixing of fluids.

Plow velocity measurements of various polar fluids near the tip of an electrified capillary were obtained by adaptive phase Doppler velocimetry. Velocities up to $3 \mathrm{~m} / \mathrm{s}$ were measured. These measurements can be used to quantify pumping of fluids through the capillary and mixing in the vicinity of the capillary by electric fields. Pressure measurements inside the capillary showed that, as the applied voltage is increased, the pressure increases with voltage, reaches a maximum, and then decreases sharply. The initial increase is due to the electric stress at the gasliquid interface, while the pressure decrease at higher voltages is due to the electrohydrodynamic flow near the tip of the capillary. The results of these studies are important in understanding the transport effects of electric fields. 


\section{SLE and SVE Studies}

These studies are planned for FY 1999.

\section{Summary of Accomplishments}

- VLE, batch-distillation, vapor-pressure, and LLE experimental arrangements have been designed and constructed to ensure safe operation for phase-equilibria studies under electric fields.

- Experimental arrangements for IR, Raman, and dielectric spectroscopy methods, including specially designed cells, a high-frequency oscilloscope, and IR and Raman spectroscopy systems, have been set up for molecular-level studies.

- VLE modification by electric fields has been found. Also, under certain conditions, electric fields were found to enhance the separation factor of VLE and the batch distillation of liquid mixtures.

- Electrohydrodynamic effects on vapor-liquid and liquid-liquid interfaces have been experimentally investigated. Simultaneous fluid spraying, pumping, and mixing of fluids by electric fields have been demonstrated. Various modes of bubble formation by electric fields have also been reported.

\section{Papers and Abstracts}

Shah, V. M., K. D. Blankenship, S. S. Laughlin, and C. Tsouris. 1997. "Distillation Under Electric Fields." Paper submitted to the Tenth Symposium on Separation Science and Technology for Energy Applications, Gatlinburg, Tenn., October 20-24, 1997.

Shah, V. M., K. D. Blankenship, S. S. Laughlin, and C. Tsouris. 1997. "New Developments in Electrodistillation." Extended abstract, submitted for AIChE Annual Meeting, Separations Division, Los Angeles, Calif., November 16-21, 1997.

Shin, W.-T, S. Yiacoumi, and C. Tsouris. 1997. "Experiments on Electrostatic Dispersion of Air in Water." Ind. Eng. Chem. Res. 36: 3647-55.

Tsouris, C. 1997. "Pumping and Mixing of Fluids by Electric Fields." Paper presented at Mixing XVI, Williamsburg, Va., June 22-27, 1997.

Tsouris, C. 1997. "Status and Directions in Electroseparations." Invited paper presented at the Chemicals and Petroleum Strategic Research Meeting of the Electric Power Research Institute (EPRI), Palo Alto, Calif., July 21-22, 1997. 
Tsouris, C., W.-T. Shin, and S. Yiacoumi. "Formation of Microbubbles Using Electric Fields for Environmental Applications." Paper presented at the 71st Colloids and Surface Science Symposium, University of Delaware, June 29-July 2, 1997. 